\title{
Prevalence of meticillin-resistant Staphylococcus aureus amongst professional meat handlers in the Netherlands, March-July 2008
}

R de Jonge (rob.de.jonge@rivm.nl) ${ }^{1}$, E Eerdier ${ }^{1,2}$, A H Havelaar ${ }^{1,3}$

1. National Institute for Public Health and the Environment, Bilthoven, The Netherlands

2. Erasmus Medical Center, Rotterdam, the Netherlands

3. Utrecht University, Utrecht, the Netherlands

Citation style for this article:

de Jonge R, Verdier JE, Havelaar AH. Prevalence of meticillin-resistant Staphylococcus aureus amongst professional meat handlers in the Netherlands, March-July 2008. Euro Surveill. 2010;15(46):pii=19712. Available online: http://www.eurosurveillance.org/ViewArticle.aspx?Articleld=19712

Article published on 18 November 2010

In the Netherlands, meticillin-resistant Staphylococcus aureus (MRSA) is detected on pork and veal farms, and hence farmers working with MRSA-positive animals are at an increased risk of being colonised. Recently retail meat products have been found positive for MRSA. Therefore, we tested the prevalence of MRSA among employees who work in the cold meat processing industry and in institutional kitchens. Nasal swabs and samples from the employees' hands as well as the handled meat were tested quantitatively and qualitatively for the presence of MRSA. Typical colonies were confirmed by PCR and typed using multi-locus sequence typing and spa-typing. All samples taken from 95 employees tested negative for MRSA, but 31 carried MSSA. From meat, five of 35 samples were positive for MRSA, containing between 0.01 and more than 10 bacteria per gram. The risk for professionals of MRSA colonisation from handling raw meat was therefore low in our setting, suggesting that the general population is at an even lower risk of being infected through meat handling.

\section{Introduction}

Staphylococcus aureus is a Gram-positive, catalasepositive commensal bacterium colonising both humans and animals. S. aureus is known for causing food poisoning through the production of enterotoxins $[1,2]$. Worldwide, strains have emerged that are resistant to a wide range of antibiotics. In the Netherlands, $0.6 \%$ of all $S$. aureus strains isolated from hospitals between 1999 and 2003 [3], were resistant to meticillin. From 2004 till 2007, this number increased to $1.1 \%$, which is still well below the average for Europe: $23.7 \%$, according to the European Antimicrobial Resistance Surveillance System (EARSS-network, now EARS-Net; [4]).

In 2004, Wertheim et al. [5] measured persons without predisposing risk to MRSA carriage before hospital admission and established that the prevalence of MRSA in the Dutch population was $0.03 \%$. Between 2002 and 2006, newly recognised infections in the Netherlands were found to be caused by a zoonotic, originally PFGE non-typable, strain of MRSA. Surveillance studies have shown that this strain, not detected before 2002, accounted for up to $5.5 \%$ of all human MRSA isolates in the first half of 2006 and up to $21 \%$ in the second half of the same year [6]. A partial explanation for this steep increase is the fact that screening of risk groups, including persons frequently in contact with pigs or calves, for MRSA carriage at hospital admission was implemented in the Netherlands in 2006. Later, the same strain was typed by multi-locus sequence typing (MLST), and it was shown that the vast majority of isolates of this strain belonged to sequence type (ST) 398 [6]. Another typing method, staphylococcal protein A (spa) typing, is frequently used to determine the number and sequence of repeats in the spa gene. MRSA ST398 from livestock animals frequently contains spa types to11, to34, t108 and t567 [9,10]. The virulence potential of MRSA is associated with its ability to produce the Panton-Valentine leukocidin (PVL) cytotoxin [9].

The first links between farming and MRSA were made in 2004 and 2005, when a farmer and his family were infected, treated for eradication of $S$. aureus, then reinfected. Later, the farmer's pigs were proven to be colonised with a genotypically indistinguishable MRSA strain $[10,11]$. In 2006, 31 pig farms were screened in the Netherlands. On seven farms, pigs were colonised with MRSA. Eleven of the 22 farmers who had undergone voluntary screening were also colonised with MRSA. All isolates were negative for PVL and not typable by PFGE (hence at the time likely to be ST398) [7]. Another notable finding from this study was that a few pigs that were negative for MRSA became MRSA carriers after treatment with oxytetracycline for respiratory problems.

Other evidence that contact with animals can lead to higher S. aureus carriage was provided by a French study in which farmers were compared to non-farmers [12]. Farmers proved to have a significantly higher 
S. aureus colonisation ratio (44.6\%) compared to nonfarmers (24.1\%). In a Dutch [6] and in a Danish casecontrol study [13], it was demonstrated that pigs were indeed a source of the rapidly emerging ST398 MRSA.

In the Netherlands, the prevalence of MRSA in animals was also estimated in slaughterhouses. A total of 540 pigs were screened, a randomised selection of ten animals from 54 batches from nine slaughterhouses. Of those, 209 animals (39\%) were positive for MRSA, distributed over $44(81 \%)$ of the batches [14]. All samples were ST398 and negative for PVL.

Given that farm animals, farmers and slaughterhouses have been found positive for MRSA, the prevalence of MRSA in meat in the Netherlands was subsequently assessed. Meat samples of various species from retail suppliers were checked for the presence of MRSA, with $11.2 \%$ testing positive. Highest prevalences were found in meat from turkeys (31.3\%), chicken $(27.3 \%)$, veal $(16.8 \%)$ and pigs (10.4\%). Of all MRSA isolates, $84 \%$ (116 of 138) belonged to ST398 [15].

Although food can be a vehicle for MRSA, the consumption of MRSA-colonised meat is thought to carry only a small risk since heating is likely to kill all bacteria and $S$. aureus is assumed to be present only on the surface of the meat. However, there might be a risk of direct transmission from raw meat [16], especially for people who work with meat intensively. Transmission has already been assessed for Micrococcus luteus, and transmission from a hamburger to hands was shown to occur, albeit at the low rate of $0.06 \%[17]$.

In this study, we assessed the risk of colonisation with both MRSA and meticillin-sensitive S. aureus (MSSA) for professionals who work intensively with raw meat products.

\section{Methods}

\section{Target population}

The study was conducted between March and July 2008. The selected population consisted of professionals who worked with raw meat on a daily basis, but were not in contact with live farm animals as part of their work. It included institutional kitchen staff (from two hospitals) and staff working at three facilities processing cold meat, where carcass parts were cut into portions for consumption. Every person in the study population had to sign an informed consent form before the start of the sampling. A questionnaire was used to assess background risks and exposure [18].

\section{Sampling of humans}

A single nasal swab (Transwab, Medical Wire and Equipment, England) was collected from each participant and stored in an ice box until further analysis on the same day for MRSA and MSSA.

To test for the presence of MRSA and MSSA on hands, we used a rinse method [19]. Participants were asked to put on a sterile nitrile glove. Sterile Mueller Hinton broth (BD, United States) with $6.5 \% \mathrm{NaCl}(\mathrm{MH}+; 30 \mathrm{ml})$ was then poured into the glove, and after 30 seconds of soaking, gloves and $\mathrm{MH}+$ were collected in a sterile stomacher bag. If the participants already wore gloves, these were collected into a stomacher bag and $30 \mathrm{ml}$ $\mathrm{MH}+$ were added. All bags were stored in an ice box for a maximum of three hours at temperatures at $2-5{ }^{\circ} \mathrm{C}$, until further analysis at the end of the day.

\section{Sampling of meat}

Meat samples were taken from a single randomly chosen piece of meat that was being prepared. Participants working with meat were asked to deposit a piece of it in a sterile bag. The samples were kept in separate containers at low temperatures (ice box, $2-5^{\circ} \mathrm{C}$ ) during transport and storage until further analysis at the end of the day.

\section{Microbiological analysis \\ Nasal swabs}

Nasal swabs were analysed for the presence of MRSA and MSSA in two ways. To detect high numbers, indicative for colonisation, the swabs were streaked directly onto a MRSA screening plate (MRSA brilliance, Oxoid) [20] and on a Baird Parker agar plate (Oxoid) supplemented with rabbit plasma fibrinogen (Oxoid). To detect low numbers, indicative for transmission, the swabs were then incubated in $10 \mathrm{ml} \mathrm{MH}+$ for 18 hours at $37^{\circ} \mathrm{C}$. For a second enrichment step, $1 \mathrm{ml}$ was transferred to $9 \mathrm{ml}$ phenol red mannitol solution with $75 \mu \mathrm{g} / \mathrm{ml}$ aztreonam (MP Biomedicals, United States) and $0.4 \mu \mathrm{g} / \mathrm{ml}$ ceftizoxime (PRM tube, Biomerieux) and incubated for

\section{TABLE 1}

Primer sequences used for typing of meticillin-resistant Staphylococcus aureus

\begin{tabular}{|l|c|c|}
\hline Gene & Primer & Sequence \\
\hline \multirow{2}{*}{ MecA [22] $]$} & MecA-1 & 5'GTTGTAGTTGTCGGGTTTGG3' \\
\cline { 2 - 3 } & MecA-C3 & 5'CTTCCACATACCATCTTCTTAAC3' \\
\hline \multirow{2}{*}{ PVL [23] } & SaPVL-1 & 5'ATCATTAGGTAAAATGTCTGGACATGATCCA3' \\
\cline { 2 - 3 } & SaPVL-2 & 5'GCATCAA(GC)TGTATTGGATAGCAAAAGC3' \\
\hline \multirow{2}{*}{$\begin{array}{l}\text { Martineau } \\
\text { [24] }\end{array}$} & Sa442-1 & 5'AATCTTGG-TCGGTACACGATATTCTCACG3' \\
\cline { 2 - 3 } & Sa442-2 & 5'CGTAATGAGATTTCAGTAGATAATACAACA3' \\
\hline
\end{tabular}

\section{TABLE 2}

Prevalence of meticillin-resistant Staphylococcus aureus on different types of meat, the Netherlands, March-July 2008 $(n=35)$

\begin{tabular}{|l|c|c|c|}
\hline \multirow{2}{*}{ Origin } & \multicolumn{2}{|c|}{ MRSA present } & Total \\
\hline Veal & Yes & No & \\
\hline Pork & 1 & 15 & 16 \\
\hline Chicken & 2 & 8 & 10 \\
\hline Turkey & 2 & 4 & 6 \\
\hline Fish & 0 & 2 & 2 \\
\hline Total & 0 & 1 & 1 \\
\hline
\end{tabular}


a further 18 hours at $37{ }^{\circ} \mathrm{C}$. A loopful (approximately $1 \mu \mathrm{L})$ of each tube was streaked on a MRSA screening plate and a Colombia agar base plate with $5 \%$ sheep blood (CAB-sb; Oxoid) and incubated for 20 to 24 hours at $37^{\circ} \mathrm{C}[20]$. Isolates were finally confirmed by PCR and typing.

Samples collected from hands and meat samples Samples were analysed as described for low numbers in nasal swabs (above) following an MPN-approach. Meat samples plus $\mathrm{MH}+$ were homogenised in a pulsifier (Microgen Bioproducts) prior to incubation.

\section{MSSA screening}

After enrichment of samples in $\mathrm{MH}+$, material from the lowest dilution of any sample was tested for MSSA as described [21].

\section{PCR testing for MRSA}

All MRSA isolates were genetically characterised by PCR specific for $S$. aureus (Martineau), the mecA gene and the PVL toxin genes (Table 1). A selection of isolates was further typed by MLST and spa-typing.

\section{Data analysis}

Prevalence data of MRSA in humans were analysed with the BINOMDIST function and Solver add-in in Microsoft Excel. Most Probable Numbers (MPN) of MRSA (and $95 \%$ confidence intervals) on food were estimated using an Excel spreadsheet based on the MPN method originally described by De Man [23,24].

\section{Results}

In this study, we examined persons working in either an institutional kitchen or at a meat processing facility, as well as meat samples. The target population originally contained 101 persons (randomly chosen). For 89 persons the results from sampling and questionnaire were available. Twelve people were excluded because they were either not present at the day of sampling $(n=2)$, or they did not come into contact with meat $(n=4)$. Six people did not provide information for the questionnaire or could not read Dutch or English.

\section{Human samples}

The male:female ratio in the study population was uneven ( $80 \%$ males). The age ranged between 26 and 56 years. Sixty-eight respondents were born in the Netherlands, while 13 were from other European countries and eight were of non-European origin. Forty-one participants kept pets, while living on a farm or keeping farm animals was rare $(n=4)$. All wore an overall when entering the butchery or kitchen. During their daily activities, 80 respondents stated they were wearing protective clothing (hairnet, gloves and overall). Nine used or had used antibiotics during the six months preceding the study. Only one of them recalled the antibiotic: amoxicillin. Eleven of the participants had been admitted to a hospital during the six months preceding the study, three of them more than once. Another per- son was hospitalised abroad. Ten persons in the study population suffered from a chronic disease.

All samples from hands and noses were negative for MRSA, but 31 participants were colonised with MSSA. Given the number of samples taken, these results imply that, with $95 \%$ confidence, the prevalence of MRSA colonisation among professional meat handlers is less than $3 \%$.

\section{Meat samples}

The results of MRSA screening of the meat samples are shown in Table 2. Of 35 meat samples, five were contaminated with MRSA: pork $(n=2)$, veal $(n=1)$ and chicken $(n=2)$. MPNs of MRSA as determined in the samples varied between 0.06 and more than 10 bacteria per gram of meat (Figure).

After PCR confirmation, MRSA-positive samples were submitted to the Dutch Reference Centre for Staphylococci for spa and MLST typing. Four isolates (one veal, two pork and one chicken) were typed as ST398 and spa-type to11, and one isolate from a chicken meat sample belonged to ST9/t1430.

\section{Discussion}

Hands and noses of all meat-handling professionals tested negative for MRSA. Among these negatively tested participants were those with a predisposing risk due to the use of antibiotics or hospital admission, as well as six persons that did not provide information for the questionnaire. Samples from these people were analysed before the results of the questionnaire were available. As all samples were negative, we did not

\section{FIGURE}

Most Probable Numbers of meticillin-resistant Staphylococcus aureus in meat samples, the Netherlands, March-July 2008 ( $\mathrm{n}=35)$

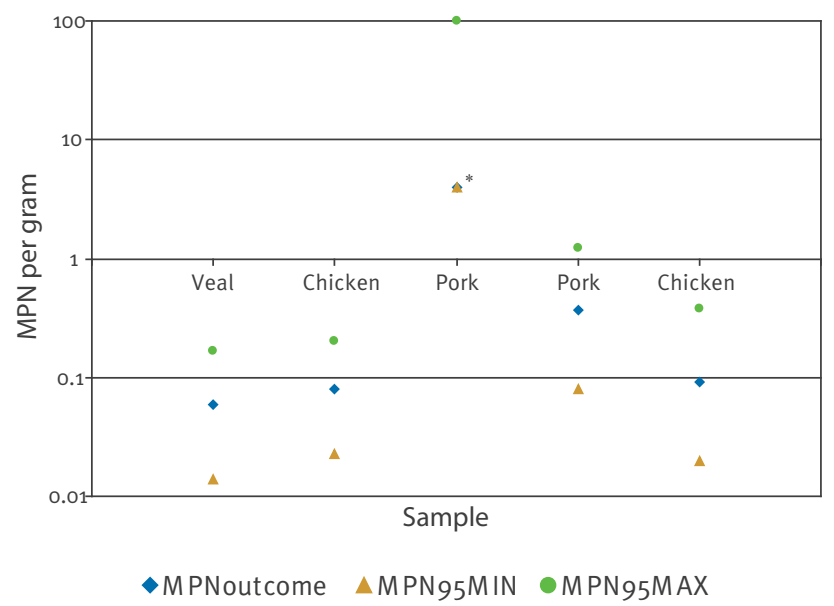

Lines indicate $95 \%$ confidence intervals, with the marker representing the Most Probable Number (MPN) per gram of sample. *All MPN series tested positive for MRSA. The MPN of this sample was at least 110 bacteria per examined sample (approximately 25 g), or four bacteria per gram. The upper $95 \%$ confidence interval limit was infinite. 
consider it necessary to exclude them from the analysis at a later stage. This result implies an upper putative colonisation rate of $3 \%$. However, the observed prevalence of $S$. aureus (MSSA) in our study, 33\%, was somewhat higher than the $24 \%$ reported for the general population in the Netherlands [5]. These results indicate that the susceptibility of our study population to $S$. aureus is at least as high as that of the general population and suggest that the observed absence of MRSA was not biased by a particular resistance of this group to $S$. aureus in general.

MRSA was found in five meat samples, with four isolates belonging to the MRSA strain ST398/to11 that has been associated with livestock [8], and one (a chicken meat sample) showing a type similar to those recently detected in chicken meat [15]. The prevalence of $14.3 \%$, MRSA in the meat samples in this study was slightly higher compared with previously published data that reported a prevalence of $11.2 \%$ [15].

In a Swiss study done in 2009, MRSA could not be detected in pig farmers and slaughterhouse employees [27]. In contrast, a Dutch study from 2010 [18] found a high prevalence of nasal MRSA carriage (5.6\%) in pigslaughterhouse workers. The difference between the two studies correlated with a difference in the prevalence of MRSA in pigs between Switzerland and the Netherlands. In the present study, we investigated the prevalence of MRSA in meat-handling professionals, as it had been reported that the prevalence of MRSA in meat was high [15]. However, we were unable to detect MRSA in our test population. This might be due to the fact that the chosen test group was too small, or to different routes transmission of MRSA in slaughterhouses and meat processing facilities. Additionally, rather than a difference in prevalence, there may have been a difference in the concentrations of MRSA in MRSApositive samples, which would result in different levels of exposure. We determined that the MPN of MRSA present in our meat samples varied between 0.01 and more than 10 per gram. For one sample, no accurate MPN could be determined as all tested dilution series were positive. Unfortunately, most studies on the prevalence of MRSA lack information on the concentration of MRSA.

This study showed that high-frequency exposure in the tested population did not result in a measurable risk of colonisation with MRSA. While the number of sampled persons, and hence the power of the study was limited, we believe that these findings imply that the risk of colonisation by contact with raw meat for the general Dutch population should be at most equal, if not several orders of magnitude lower. Professional meat handlers come into contact with raw meat many times every day, whereas the general public would come into contact with raw meat once a day or more rarely. The upper limit of colonisation prevalence of professionals (3\%) would therefore correspond to a much lower estimate for the general population, which is in agreement with a prevalence of $0.03 \%$ in the Dutch population as reported by Wertheim et al. [5].

\section{Acknowledgements}

We like to thank Arjen van de Giessen and Xander Huijsdens for helpful suggestions and critically reading the manuscript, and Paul Hengeveld and Thijs Bosch for technical assistance.

References

1. Dack GM, Cary WE, Woolpert O, Wiggers H. An outbreak of food poisoning proved to be due to a yellow hemolytic Staphylococcus. J Prevent Med. 1930;4:167-75.

2. The microbiological monitoring of foods. In: Mossel DAA, Corry JEL, Struijk CB, Baird RM, editors. Essentials of the microbiology of foods. Chichester: John Wiley and Sons Ltd, 1995. P. 319.

3. Tiemersma EW, Bronzwaer SL, Lyytikäinen O, Degener JE, Schrijnemakers P, Bruinsma N, et al. Methicillin-resistant Staphylococcus aureus in Europe, 1999-2002. Emerg Infect Dis. 2004;10(9):1627-34.

4. EARSS Annual report 2007. Bilthoven: European Antimicrobial Resistance Surveillance System; October 2008. ISBN: 978-90 6960-214-1. Available from: http://www.ecdc.europa.eu/en/ activities/surveillance/EARS-Net/Documents/2007_EARSS_ Annual_Report.pdf

5. Wertheim HF, Vos MC, Boelens HA, Voss A, VandenbrouckeGrauls CM, Meester $\mathrm{MH}$, et al. Low prevalence of methicillinresistant Staphylococcus aureus (MRSA) at hospital admission in the Netherlands: the value of search and destroy and restrictive antibiotic use. J Hosp Infect. 2004;56(4):321-5.

6. van Loo I, Huijsdens X, Tiemersma E, de Neeling A, van de Sande-Bruinsma N, Beaujean D, et al. Emergence of methicillin-resistant Staphylococcus aureus of animal origin in humans. Emerg Infect Dis. 2007;13(12):1934-9.

7. van Duijkeren E, Ikawaty R, Broekhuizen-Stins MJ, Jansen MD, Spalburg EC, de Neeling AJ, et al. Transmission of methicillinresistant Staphylococcus aureus strains between different kinds of pig farms. Vet Microbiol. 2008;126(4):383-9.

8. van Cleef BA, Verkade EJM, Wulf MW, Buiting AG, Voss A, Huijsdens XW, et al, 2010. Prevalence of livestockassociated MRSA in communities with high pig densities in the Netherlands. PLoS One. 5(2):e9385. doi:10.1371/journal. pone. 0009385 .

9. Welinder-Olsson C, Florén-Johansson K, Larsson L, Oberg S, Karlsson L, Ahrén C. Infection with Panton-Valentine leukocidin-positive methicillin-resistant Staphylococcus aureus to34. Emerg Infect Dis. 2008;14(8):1271-2.

10. Huijsdens XW, van Dijke BJ, Spalburg E, van Santen-Verheuve MG, Heck ME, Pluister GN, et al. Community-acquired MRSA and pig-farming. Ann Clin Microbiol Antimicrob. 2006;5:26.

11. Voss A, Loeffen F, Bakker J, Klaassen C, Wulf M. Methicillinresistant Staphylococcus aureus in pig farming. Emerg Infect Dis. 2005; 11(12):1965-6.

12. Armand-Lefevre L, Ruimy R, Andremont A. Clonal comparison of Staphylococcus aureus isolates from healthy pig farmers, human controls, and pigs. Emerg Infect Dis. 2005;11(5):711-4.

13. Lewis HC, Mølbak K, Reese C, Aarestrup FM, Selchau M, Sørum, et al. Pigs as a source of methicillin resistant Staphylococcus aureus CC398 infections in humans, Denmark. Emerg Infect Dis. 2008;14(9):1383-9.

14. de Neeling AJ, van den Broek MJ, Spalburg EC, van SantenVerheuvel MG, Dam-Deisz WD, Boshuizen HC, et al. High prevalence of methicillin resistant Staphylococcus aureus in pigs. Vet Microbiol. 2007;122(3-4):366-72.

15. de Boer E, Zwartkruis-Nahuis JT, Wit B, Huijsdens XW, de Neeling AJ, Bosch T, et al. Prevalence of methicillinresistant Staphylococcus aureus in meat. Int J Food Protect. 2009;134(1-2):52-6.

16. Scientific Opinion of the Panel on Biological Hazards on a request from the European Commission on Assessment of the Public Health significance of meticillin resistant Staphylococcus aureus (MRSA) in animals and foods. The EFSA Journal. 2009;993:1-73.

17. Rusin P, Maxwell S, Gerba C. Comparative surface-to-hand and fingertip-to-mouth transfer efficiency of Gram-positive bacteria, Gram-negative bacteria, and phages. J Appl Microbiol. 2002;93(4):585-92. 
18. Van Cleef BA, Broens EM, Voss A, Huijsdens XW, Züchner Van Benthem BH, et al. High prevalence of nasalMRSA carriage in slaughterhouse workers in contact with live pigs in the Netherlands. Epidemiol Infect. 2010;138(5):756-63.

19. Onderzoek van handen. [Examination of hands]. In: Dijk R, Grootenhuis A, editors. Microbiologie van voedingsmiddelen: methoden, principes en criteria. [Microbiology of food: methods, principles and criteria]. Houten: Noordervliet Media BV; 2007 . Dutch. ISBN 9789072072771.

20. Verkade E, Elberts S, Verhulst C, Kluytmans J. Performance of Oxoid Brilliance MRSA medium for detection of methicillinresistant Staphylococcus aureus: an in vitro study. Eur J Clin Microbiol Infect Dis. 2009;28(12):1443-6.

21. Microbiology of food and animal feeding stuffs - Horizontal method for the enumeration of coagulase-positive staphylococci (Staphylococcus aureus and other species). Part 2: Technique using rabbit plasma fibrinogen agar medium. 1st ed. Geneva: International Organization for Standardization; 1999. ISO 6888-2:1999.

22. de Neeling AJ, van Leeuwen WJ, Schouls LM, Schot CS, van Veen-Rutgers A, Beunders AJ, et al. Resistance of staphylococci in The Netherlands: surveillance by an electronic network during 1989-1995. J Antimicrob Chemother. 1998;41(1):93-101.

23. Lina G, Piémont Y, Godail-Gamot F, Bes M, Peter MO, Gauduchon V, et al. Involvement of Panton-Valentine leukocidin-producing Staphylococcus aureus in primary skin infections and pneumonia. Clinlnfect Diseas. 1999;29(5):1128-1132.

24. Martineau F, Picard FJ, Roy PH, Ouellette M, Bergeron MG. Species-specific and ubiquitous-DNA-based assays for rapid identification of Staphylococcus aureus. J Clin Microbiol. 1998;36(3):618-23.

25. de Man JC. MPN Tables, corrected. Eur J Appl Microbiol Biotechnol. 1983;17(5):301-5.

26. de Man JC. MPN Tables for more than one test. Eur J Appl Microbiol. 1977;4(4):307-16.

27. Huber H, Koller S, Giezendanner N, Stephan R, Zweifel C. Prevalence and characteristics of meticillin-resistant Staphylococcus aureus in humans in contact with farm animals, in livestock, and in food of animal origin, Switzerland, 2009. Euro Surveill. 2010;15 (16): pii=19542. Available from: http://www.eurosurveillance.org/ViewArticle. aspx?Articleld $=19542$ 